\title{
When and How to Extubate Premature Infants from Mechanical Ventilation
}

\author{
Wissam Shalish • Guilherme Mendes Sant' Anna • \\ Girija Natarajan · Sanjay Chawla
}

Published online: 29 October 2013

(C) Springer Science + Business Media New York 2013

\begin{abstract}
Prolonged mechanical ventilation (MV) is associated with both short-term morbidities and neurodevelopmental impairment. The ability of clinicians to accurately predict successful extubation is limited. Extubation failure may also be associated with significant shortand long-term morbidities. There are limited conflicting data from a few studies evaluating the role of pulmonary function and autonomic nervous system function in predicting the success of elective extubations. Also, the methodology used in some studies may not be easily applied in the clinical setting. There is a need to identify good objective criteria for predicting successful extubation, which may help reduce the duration of MV as well as the incidence of failed extubation.
\end{abstract}

Keywords Mechanical ventilation - Extubation . Premature

W. Shalish · G. M. Sant' Anna

Division of Neonatology, Montreal Children's Hospital, McGill

University, Montreal, QC, Canada

e-mail: wissam.shalish@mail.mcgill.ca

G. M. Sant' Anna

e-mail: guilherme.santanna@mcgill.ca

G. Natarajan $\cdot$ S. Chawla

Division of Neonatal Perinatal Medicine, Hutzel Women's

Hospital, Wayne State University, Detroit, MI, USA

e-mail: gnatara@med.wayne.edu

G. Natarajan · S. Chawla $(\bowtie)$

Division of Neonatology, Children's Hospital of Michigan,

3901 Beaubien, Detroit, MI 48201, USA

e-mail: schawla@dmc.org

\section{Introduction}

Many extreme preterm infants (gestational age $\leq 28$ weeks) are unable to sustain adequate oxygenation and ventilation without the support of mechanical ventilation (MV). In a large multicenter cohort study $(n=5,364), 89 \%$ of the extremely low birth weight (ELBW) infants required MV during the first day of life [1]. The coexistence of lung immaturity, weak respiratory drive and surfactant deficiency often contributes to this dependency on MV during the first days of life. Unfortunately, the need for MV is associated with increased mortality and morbidities such as ventilatorassociated pneumonia, pneumothorax and bronchopulmonary dysplasia [2, 3]. Furthermore, each additional week of MV is associated with a significant increase in neurodevelopmental impairment [1]. Thus, clinicians have considered extubation of preterm infants as early as possible a goal of care.

The disconnection of a patient from MV is a complex and continuous process that involves active weaning of the ventilatory settings, evaluation of extubation readiness, and provision of adequate supervision and non-invasive support following disconnection. There are three important shortfalls in our understanding of this process in this vulnerable group. First, the methods of weaning from the ventilator and proceeding toward extubation have been variable and inconsistent across neonatal units. Second, our ability to accurately predict which infants are ready for extubation has been suboptimal. Finally, the ideal method of noninvasive support is still under investigation. For these reasons, extreme preterm infants have the highest rates of extubation failure, with up to $50 \%$ of ELBW infants failing extubation in some centers [4-6].

Extubation failure is a significant clinical problem. The prevalence of extubation failure is quite difficult to determine since the criteria used for re-intubation and 
definitions of failure are diverse. Common reasons for reintubation include severe or frequent apneas, hypoxemia, increased work of breathing and significant respiratory acidosis [7]. The majority of recent studies have defined extubation failure as the need for re-intubation within $72 \mathrm{~h}$ of extubation, but this definition is empirical and lacks strong evidence to support it [6, 8, 9].

Extubation failure has been independently associated with increased mortality rates, longer length of hospital stay, and more days on oxygen and ventilatory support [9-11]. In the short-term, endotracheal reintubation is not an easy and benign process. The proficiency in intubation of premature neonates has declined over the last years, with recent studies reporting successful rates between 60 and $73 \%$, with a long time needed for intubation [12, 13]. Endotracheal intubation may further be associated with discomfort to the patient, malposition of the tube, trauma to the airway and hemodynamic instability [12, 14-17]. It has even been associated with alterations in brain function, as monitored by EEG [18].

Thus, the current literature clearly supports that both prolonged ventilation and premature unsuccessful extubation followed by reintubation are associated with poor outcomes. Therefore, determining the optimal time and best strategy for successful weaning and extubation of ventilated premature infants is crucial to reduce unwarranted complications.

\section{When to Extubate}

Neonatologists should start to consider weaning the levels of ventilatory support as soon as the extreme preterm infant is intubated. It is critical that a proactive weaning process is in place and followed. This will allow for the provision of minimal necessary support, while reducing lung injury. Preterm infants should then be considered for extubation when at minimal ventilatory settings and deemed at the highest chance of a successful attempt. Unfortunately, the ability to determine this has proven to be very difficult. In the absence of specific protocols, weaning from MV has been highly dependent on the physician's expertise and past experiences. Furthermore, the rates of weaning ventilatory parameters and the decision to extubate is still based on clinical evaluation, blood gases, oxygen needs and level of ventilatory support [8]. In the absence of objective, evidence-based criteria, there is still no consensus on the ventilator settings at which a preterm infant should be considered for extubation with a high likelihood of success.

\section{Use of Weaning Protocols}

There is salient evidence that ventilation protocols improve outcomes, reduce costs, and decrease the duration of MV and length of stay in adult intensive care units [19-21]. The Cochrane systematic review of three trials in critically ill pediatric patients suggested that protocolized weaning from the ventilator reduced the duration of MV but had no significant effects on length of stay in the hospital or pediatric intensive care unit, mortality or adverse events [22]. In contrast, there is a paucity of data on the role of weaning protocols in the neonatal population. A single observational study from Canada noted that implementation of a protocol for weaning and extubation of premature infants resulted in improved outcomes, earlier extubation and an increase in the success of extubation [23•].

\section{Pre-extubation Ventilation Strategies}

With the rapid advancement of technology, neonatologists are faced with the difficult task of choosing the best ventilator mode for their patients. Popular modes of ventilation include assist control (A/C), synchronized intermittent mandatory ventilation (SIMV) and high-frequency oscillatory ventilation (HFOV). When compared with SIMV, A/C appears to provide more homogeneous tidal volumes, less work of breathing and more rapid weaning from MV $[24,25]$. However, the combination of SIMV with pressure support (PS) circumvents the resistance of the endotracheal tube and may potentially be comparable to $\mathrm{A} / \mathrm{C}$ in weaning toward extubation [26]. Another attractive ventilation strategy has been the use of volume-controlled ventilation, as opposed to pressure-limited ventilation, in weaning preterm infants. In this mode, weaning occurs automatically as the patient's lung compliance improves. Volumetargeted ventilation reduces the risk of barotrauma and ventilator-induced lung injury, and it has been associated with lower rates of death, BPD and shorter durations of MV [27]. Direct extubation of preterm infants from HFOV is also practiced in some centers, although no study has looked at benefits incurred from this approach. In a recent review article, Sant'Anna and Keszler [7] proposed guidelines of ventilatory settings from which preterm infants (younger than 2 weeks) may be extubated, according to the ventilation mode used.

\section{Permissive Hypercapnia}

In conjunction with volume-targeted ventilation, permissive hypercapnia is a strategy that may facilitate weaning from MV and reduce ventilator-induced lung injury. Various $\mathrm{PaCO}_{2}$ targets have been used in studies, although generally they are limited by a pH of at least 7.20-7.25. Preterm infants exposed to permissive hypercapnia for 10 days or longer have shown improved respiratory drive, a reduction in number of days on $\mathrm{MV}$, and a trend toward reduction of BPD or death [28]. This effect has not been 
observed when the intervention was done in the first 4 postnatal days. Moreover, the safe limits of $\mathrm{PaCO}_{2}$ in the first few days of life may be very different than during the chronic phase of BPD. For this reason, more research is necessary to elucidate the optimal target $\mathrm{CO}_{2}$ levels and durations for optimizing the strategy of permissive hypercapnia. In the meantime, it appears suitable to target $\mathrm{PaCO}_{2}$ levels of 50-60 while maintaining a $\mathrm{pH}$ over 7.20.

\section{Oxygen Saturation Ranges}

Oxygen supplementation is very commonly provided to extremely preterm infants during MV. Despite the recently heightened interest in the subject, our current knowledge of the optimal range of oxygen saturation $\left(\mathrm{SpO}_{2}\right)$ in this vulnerable population remains limited. From the current available literature, it appears that both very high (95-98 \%) and very low (83-87\%) $\mathrm{SpO}_{2}$ ranges may be harmful to the preterm infant. In the benefit of oxygen saturation targeting (BOOST) trial, infants assigned to the high saturation group (95-98\%) had higher $\mathrm{O}_{2}$ dependency at 36 weeks post-conceptual age and higher home $\mathrm{O}_{2}$ therapy compared to their peers in the 91-94\% $\mathrm{SpO}_{2}$ range [29]. There were no conferred benefits from highsaturation on growth or neurodevelopmental measures. Similar results were obtained in the supplemental therapeutic oxygen for pre-threshold retinopathy of prematurity (STOP-ROP) trial, whereby infants exposed to the higher saturation targets $\left(\mathrm{SpO}_{2} 96-99 \%\right)$ had longer hospitalizations as well as prolonged oxygen and ventilator dependence [30]. In the surfactant, positive pressure and pulse oximetry randomized (SUPPORT) trial, infants in the lower $\mathrm{SpO}_{2}$ range (85-89\%) had significantly lower rates of severe retinopathy among survivors. However, there was also a higher mortality rate overall, which raised serious concerns about the safety of this practice [31]. The more recent BOOST II Trial (UK, Australia, New Zealand) also showed increased mortality in the 85-89\% saturation group during an interim safety analysis, which prompted earlier termination of the study [32]. Interestingly, the latest Canadian oxygen trial (COT) did not report any differences in mortality between high- and low-saturation groups [33]. A meta-analysis is currently underway, by the Neonatal Oxygenation Prospective Meta-analysis Collaboration, to analyze individual patient data from all of the aforementioned trials [34]. In the meantime, it appears prudent to keep $\mathrm{SpO}_{2}$ between 91 and $95 \%$ in extremely preterm infants until more data are available.

Postnatal Corticosteroids

Extremely preterm infants who are on prolonged MV (beyond 7-14 days) are at high risk of developing BPD.
Systemic corticosteroids can be very effective in the process of weaning from MV and in the treatment of BPD. However, their routine use has fallen out of favor since recognition of their potential harms on neurodevelopmental outcomes [35]. These harms were mainly found in studies where corticosteroids were given in the first week of life, at very high doses and for protracted periods. In fact, the American Academy of Pediatrics Committee on Fetus and Newborn published a policy statement in 2010 stating that low-dose dexamethasone or low-dose hydrocortisone may facilitate extubation and decrease the incidence of BPD without affecting long-term neurodevelopmental outcomes $[36,37]$. Notwithstanding, their routine is still not recommended. For patients with a history of airway edema due to multiple intubations, traumatic intubations or prolonged intubations, a short course of dexamethasone in the periextubation period may facilitate successful extubation. For this reason, clinicians should balance the risks of BPD with the potential adverse effects of postnatal steroids on an individual basis, in conjunction with the infant's parents. According to the current literature, it is preferable to administer a short course of dexamethasone at a starting dose of approximately $0.15 \mathrm{mg} / \mathrm{kg} /$ day or hydrocortisone at a starting dose of approximately $5 \mathrm{mg} / \mathrm{kg} /$ day.

\section{Predictors of Successful Extubation}

Once the preterm infant has achieved ventilatory settings when extubation seems to be a reasonable next step, physicians should evaluate whether the patient is 'ready' or not for extubation. This is a very important phase of this whole process since some patients can be easily weaned to low settings but are not yet prepared to cope with respiration without invasive support. The following section will review the role of clinical factors, pulmonary function tests, spontaneous breathing trials (SBT) and autonomic nervous system function evaluation in assessing extubation readiness in extremely preterm infants.

\section{Clinical Factors}

Several epidemiological studies have tried to identify the risk factors associated with extubation failure in preterm infants. Those include low gestational age ( $<26$ weeks), prolonged ventilation ( $>10-14$ days), low $\mathrm{pH}$ and extubation from high ventilatory settings (e.g., high mean airway pressure and high $\left.\mathrm{FiO}_{2}\right)[6,7,9]$. Infants with a history of previous extubation failure or multiple intubation attempts are also predisposed to fail extubation again. In addition, the presence of other morbidities, such as residual lung disease (e.g., bronchopulmonary dysplasia) or a hemodynamically significant patent ductus arteriosus (PDA) can 
increase the likelihood of extubation failure [7]. Recent attempts have been made to create automated prediction models, using machine learning approaches to aid clinicians in making an objective decision about optimal extubation time [38, 39]. These methods, although very attractive, require further prospective clinical validation.

\section{Pulmonary Function Tests}

Data on pulmonary function testing prior to extubation of preterm infants are limited and conflicting [8, 9, 40-46]. Some of the variables that have been investigated include tidal volume, minute ventilation (MV), functional residual capacity (FRC), compliance and airway resistance. Most studies have a small sample size, comprise a wide range of gestational ages and often fail to control for confounders such as infection, lung disease and PDA. Moreover, they were done at a time when antenatal corticosteroids, surfactant and gentler ventilation strategies were still not part of routine practice. For instance, Szymankiewicz and Balsan [41, 42] noted that higher lung compliance and lower airway resistance measured prior to extubation of very low birth weight infants were predictive of successful extubation. However, these studies did not control for surfactant, antenatal steroids or caffeine, and most infants did not receive continuous positive airway pressure (CPAP) consistently after extubation. In contrast, VenessMeeham and Chawla $[9,40]$ showed that lung compliance was a poor predictor of successful extubation in preterm infants with RDS. Other studies, more reflective of current practices, have shown some merit to measurements of MV as well as respiratory and diaphragmatic pressure-time indices to assess respiratory muscle strength and diaphragmatic efficiency, respectively [44-46]. Nonetheless, these tests have only been evaluated in a small sample population and would need to be prospectively reproduced on a much larger scale.

\section{Spontaneous Breathing Trials}

SBTs have been validated and extensively used in adult and pediatric patients as a means of predicting successful extubation [47-49]. In premature infants, though, data to justify their use are insufficient. A meta-analysis on SBT concluded that preterm infants should be extubated directly without a trial of SBT [50]. However, the meta-analysis included old studies ( $>20$ years old) in which ventilation strategies were very different. Furthermore, the duration of spontaneous breathing was long (ranging from 6 to $24 \mathrm{~h}$ ) and the pressures used during endotracheal CPAP were low (2-4 $\mathrm{cm} \mathrm{H}_{2} \mathrm{O}$ ) [51-53].

A number of more recent studies have redefined the role of SBTs. Gillespie and colleagues conducted a small randomized controlled trial $(n=42)$ assessing the performance of a spontaneous MV test in neonates with respiratory distress syndrome. Infants were placed on ETTCPAP (3-4 cm $\mathrm{H}_{2} \mathrm{O}$ ) for 10 min while spontaneous MV was measured. The MV test was considered successful when the ratio of spontaneous to mechanical MV exceeded $50 \%$. Infants evaluated by the MV test were extubated in a significantly shorter period of time (mean of $8 \mathrm{~h}$ ) compared to those evaluated clinically (mean of $36 \mathrm{~h}$ ), with a positive predictive value of $76 \%$ [44]. Vento and colleagues enrolled 41 ELBW infants and performed a 2-h endotracheal CPAP trial. They noted that the mean value of spontaneous expiratory minute volume was significantly lower in the failure group than in the successful group [45]. Kamlin et al. performed a 3-min endotracheal CPAP trial in premature infants (birth weight $<1,250 \mathrm{~g}, n=50$ ) prior to extubation. Failure of the test was defined as a desaturation or bradycardia for longer than $15 \mathrm{~s}$ despite a $15 \%$ increment in $\mathrm{FiO}_{2}$. This test showed great sensitivity, with positive and negative predictive values of 93 and $89 \%$, respectively [54]. Finally, Chawla et al. performed a 5-min SBT in 49 premature neonates ( $<32$ weeks) who were less than 3 weeks of age. The SBT was classified as failed if the patient met any one of the following: significant bradycardia (heart rate $<100$ beats/min for more than $10 \mathrm{~s}$ ), oxygen desaturation ( $<85 \%$ for $>15 \mathrm{~s}$ ) or significant bradycardia requiring intervention. The test showed excellent sensitivity and positive predictive values $(88 \%)$, but the specificity and negative predictive values were low [9].

In a prospective observational cohort study among 122 ventilated adults, three clinical variables predicted reintubation despite a successful SBT: moderate to copious endotracheal secretions $(p=0.001)$, Glasgow Coma Scale score $\leq 10 \quad(p=0.004), \quad$ and hypercapnia $\quad\left(\mathrm{PaCO}_{2}\right)$ $\geq 44 \mathrm{~mm} \mathrm{Hg}$ during the spontaneous breathing trial $(p=0.001)$ [55].

The latest studies clearly show the promise of SBTs, which can be easily performed at the bedside, in predicting extubation readiness of preterm infants. But much debate still remains around the optimal duration of the SBT, the CPAP level employed and the criteria to define failure of the test. A larger randomized trial would be warranted to better answer these questions.

\section{Autonomic Nervous System Function}

Variations in heart rate and respiratory rate are well known to be influenced by the autonomic nervous system (ANS). In fact, integrity of the cardiovascular system depends on the right balance between sympathetic and parasympathetic tones [56]. Autonomic dysfunction, as characterized by reduced heart rate variability (HRV), has been linked to increased mortality and cardiovascular disease in adult 
individuals [57]. Respiratory variability (RV), on the other hand, is reduced in conditions of hypoxia, hypercapnia and inspiratory mechanical loading (elastic or resistive) [5861]. The adult literature has consistently demonstrated reduced HRV and RV in adult patients who fail weaning from MV [62, 63].

The role of HRV and RV in predicting disease in newborn infants is not as well understood. It has however gained popularity over the past years, especially with the latest evidence that loss of HRV precedes the clinical presentation of late-onset neonatal sepsis [64]. With regards to prediction of extubation readiness, a recent small prospective observational study of 56 preterm infants (birth weight $\leq 1,250 \mathrm{~g}$ ) showed that HRV was significantly decreased in those who failed extubation [65•*]. Another study evaluated the performance of SBT and RV for prediction of extubation readiness. The combined effect of both parameters predicted successful extubation more accurately than either parameter alone [66••].

Measurement of ANS function appears to be of great future promise for predicting extubation readiness in preterm infants. Ideally, the technology should be easy to use by clinicians at the bedside, without arduous analysis. The development of automated machine learning methods to facilitate interpretation of the results would be helpful.

\section{How to Extubate}

Once an infant is deemed ready for extubation, a number of steps need to be followed methodically to ensure a successful transition. The process of extubation takes time, meticulous planning and requires the full collaboration of all team members caring for the infant (including clinicians, nurses and respiratory therapists). The procedure itself should be done in a controlled environment, in the presence of personnel skilled in respiratory stabilization and intubation (if needed). The following section will review some of the evidence-based strategies to improve extubation success in preterm infants (summary in Table 1).

\section{Use of Caffeine Prior to Extubation}

The benefits of caffeine for extremely preterm infants have been suggested in several small single-center studies and confirmed by a large multi-center trial conducted in 2006 (CAP trial) [67]. This randomized controlled trial showed that infants assigned to the caffeine group had less BPD, a shorter duration on supplemental oxygen and were intubated for almost 1 week less. A secondary, post hoc subgroup analysis showed that early administration of caffeine (before 3 days of life) could further reduce the time on MV [68•]. From these studies, we conclude that caffeine is
Table 1 Evidence-based strategies for achieving a successful extubation in extremely preterm infants ( $\leq 28$ weeks)

\footnotetext{
1. Develop and implement a mechanical ventilation protocol, including the weaning phase, extubation and reintubation criteria. This should involve all health care professionals (neonatologists, nurses and respiratory therapists) working at the NICU

2. Use assist control mode with volume-targeted ventilation if equipped with the proximal flow sensor

3. Allow for permissive hypercapnia $\left(\mathrm{PaCO}_{2} 50-60 \mathrm{mmHg}\right)$ and establish oxygen saturation targets $\left(\mathrm{SpO}_{2} 91-95 \%\right)$

4. Administer a loading dose of caffeine before extubation (ideally within $24 \mathrm{~h}$ prior to extubation)

5. Place the infant on CPAP or NIPPV immediately after extubation (PEEP $\geq 5 \mathrm{cmH}_{2} \mathrm{O}$ ) using bi-nasal prongs or a nasal mask
}

beneficial and should be part of the regimen when preparing an infant for extubation. It appears reasonable to give it within at least $24 \mathrm{~h}$ from the projected time of extubation. However, although tempting, the routine administration of caffeine immediately after birth (or in the first few hours after birth) has not been investigated.

\section{Post-extubation Ventilation Strategies}

Following extubation, premature infants still have insufficient respiratory drive and muscle strength to maintain FRC independently. For this reason, a distending pressure is necessary to reduce the risk of post-extubation atelectasis. This pressure should be applied immediately after the removal of the endotracheal tube and may be provided in the form of CPAP or nasal intermittent positive pressure ventilation (NIPPV) using short, bi-nasal prongs or nasal masks. Among 1,009 preterm infants randomly assigned to either NIPPV or nasal CPAP — at the time of the first use of noninvasive respiratory support during the first 28 days of life-rates of death or BPD and reintubation after randomization were comparable [69••].

The use of heated humidified high-flow nasal cannula (HHHFNC) post-extubation has gained popularity over the past few years. Two RCTs have evaluated the use of HHHFNC in preventing extubation failure compared to nasal CPAP in preterm infants and have showed HHHFNC to be as effective and safe as CPAP [70•, 71••]. However, only one of those studies looked specifically at the extremely preterm population ( $\leq 28$ weeks gestation). For infants $<26$ weeks of gestation at birth, no conclusion could be inferred [71••].

\section{Treatment of Postextubation Stridor}

Postextubation stridor is a relatively common occurrence that could increase the risk of extubation failure among 
extremely preterm infants. Risk factors include prolonged $\mathrm{MV}$, a history of multiple traumatic intubations or a previous failed extubation due to subglottic edema. Although broadly used, the role of postnatal steroids for the treatment and prevention of postextubation stridor remains unclear. A meta-analysis in 2009 failed to show any benefits of corticosteroids on the prevention or treatment of postextubation stridor [72]. However, in one of the studies targeting high-risk infants only, the incidence of stridor was significantly reduced when multiple doses of steroids were given around the time of extubation [73]. Therefore, it appears reasonable to reserve this therapy for high-risk infants only, until more evidence is available.

\section{Tracheostomy}

There is a paucity of literature on the indications and timing of tracheostomy in preterm infants who fail multiple extubation attempts or cannot be weaned from MV. The overall rate of neonatal tracheostomy ranges between 0.55 and $2.7 \%$, with even higher rates in the extreme low birth weight population [74]. In practice, these infants are submitted to a tracheostomy if they remain ventilatordependent by a corrected gestational age near term $( \pm$ month of life). A tracheostomy is generally performed in the hopes of effectively decreasing the need for invasive respiratory support, improving oral-motor skills and childhood lung function, improving neurodevelopmental outcomes or simply expediting discharge home. This high variability in desired objectives of tracheostomy makes it very difficult to propose any specific recommendations about its use.

\section{Conclusion}

Determining when and how extremely preterm infants can be extubated continues to be challenging. Development of clinical guidelines based on clinical characteristics of the patient along with combinations of SBT, pulmonary function tests and autonomic nervous system function will help clinicians better determine the optimal timing for elective extubation. Moreover, the development of protocols for weaning and extubating preterm infants, as well as post-extubation management and adjunctive therapies may prove to be beneficial in achieving successful elective extubations.

Disclosures Wissam Shalish, Guilherme Mendes Sant' Anna, Girija Natarajan and Sanjay Chawla declare that they have no conflict of interest.
Human and Animal Rights and Informed Consent This article does not contain any studies with human or animal subjects performed by any of the authors.

\section{References}

Recently published papers of particular interest have been highlighted as:

- Of importance,

•- Of major importance

1. Walsh MC, et al. Extremely low birthweight neonates with protracted ventilation: mortality and 18-month neurodevelopmental outcomes. J Pediatr. 2005;146:798-804. doi:10.1016/j.jpeds. 2005.01.047.

2. Bancalari E, Gerhardt T. Bronchopulmonary dysplasia. Pediatr Clin North Am. 1986;33:1-23.

3. Strong RM, Passy V. Endotracheal intubation: complications in neonates. Arch Otolaryngol. 1977;103:329-35.

4. Stefanescu BM, et al. A randomized, controlled trial comparing two different continuous positive airway pressure systems for the successful extubation of extremely low birth weight infants. Pediatrics. 2003;112:1031-8.

5. Davis PG, Henderson-Smart DJ. Nasal continuous positive airways pressure immediately after extubation for preventing morbidity in preterm infants. Cochrane Database Syst Rev. 2003;3:CD000143. doi:10.1002/14651858.CD000143.

6. Hermeto F, Martins BM, Ramos JR, Bhering CA, Sant'Anna GM. Incidence and main risk factors associated with extubation failure in newborns with birth weight $<1,250$ grams. J Pediatr. 2009;85:397-402. doi:10.2223/JPED.1922.

7. Sant'Anna GM, Keszler M. Weaning infants from mechanical ventilation. Clin Perinatol. 2012;39:543-62. doi:10.1016/j.clp. 2012.06.003.

8. Kamlin CO, Davis PG, Morley CJ. Predicting successful extubation of very low birthweight infants: Arch Dis Child. 2006;91:F180-3. doi:10.1136/adc.2005.081083.

9. Chawla S, Natarajan G, Gelmini M, Kazzi SN. Role of spontaneous breathing trial in predicting successful extubation in premature infants. Pediatr Pulmonol. 2013;48:443-8. doi:10.1002/ ppul.22623.

10. Epstein SK, Ciubotaru RL, Wong JB. Effect of failed extubation on the outcome of mechanical ventilation. Chest. 1997;112: 186-92.

11. Baisch SD, Wheeler WB, Kurachek SC, Cornfield DN. Extubation failure in pediatric intensive care incidence and outcomes. Pediatr Crit Care. 2005;6:312-8. doi:10.1097/01.PCC.00001611 19.05076.91.

12. Bismilla Z, et al. Failure of pediatric and neonatal trainees to meet Canadian neonatal resuscitation program standards for neonatal intubation. J Perinatol. 2010;30:182-7.

13. Kamlin $\mathrm{CO}$, et al. A randomized trial of stylets for intubating newborn infants. Pediatrics. 2013;131:e198-205. doi:10.1542/ peds.2012-0802.

14. Walner DL, Loewen MS, Kimura RE. Neonatal subglottic stenosis: incidence and trends. The Laryngoscope. 2001;111:48-51. doi:10.1097/00005537-200101000-00009.

15. Venkatesh V, et al. Endotracheal intubation in a neonatal population remains associated with a high risk of adverse events. Eur J Pediatr. 2011;170:223-7. doi:10.1007/s00431-010-1290-8. 
16. Schuman TA, Jacobs B, Walsh W, Goudy SL. Iatrogenic perinatal pharyngoesophageal injury: a disease of prematurity. Int $\mathbf{J}$ Pediatr Otorhinolaryngol. 2010;74:393-7. doi:10.1016/j.ijporl. 2010.01.011.

17. Schedlbauer EM, Todt I, Ernst A, Seidl RO. Iatrogenic tracheal rupture in children: a retrospective study. The Laryngoscope. 2009;119:571-5. doi:10.1002/lary.20107.

18. Shangle CE, Haas RH, Vaida F, Rich WD, Finer NN. Effects of endotracheal intubation and surfactant on a 3-channel neonatal electroencephalogram. J Pediatr. 2012;161:252-7. doi:10.1016/j. jpeds.2012.02.014.

19. Blackwood B, et al. Use of weaning protocols for reducing duration of mechanical ventilation in critically ill adult patients: Cochrane systematic review and meta-analysis. $\mathrm{Br}$ Med $\mathrm{J}$. 2011;342:7237. doi:10.1136/bmj.c7237.

20. Danckers M, et al. Nurse-driven, protocol-directed weaning from mechanical ventilation improves clinical outcomes and is well accepted by intensive care unit physicians. J Crit Care. 2013;28:433-41. doi:10.1016/j.jcrc.2012.10.012.

21. Gupta P, Giehler K, Walters RW, Meyerink K, Modrykamien AM. The effect of a mechanical ventilation discontinuation protocol in patients with simple and difficult weaning: impact on clinical outcomes. Respir Care; 2013. doi:10.4187/respcare.02558.

22. Blackwood B, Murray M, Chisakuta A, Cardwell CR, O'Halloran P. Protocolized versus non-protocolized weaning for reducing the duration of invasive mechanical ventilation in critically ill paediatric patients. Cochrane Database Syst Rev. 2013;7:CD009082. doi:10.1002/14651858.CD009082.pub2.

23. - Hermeto F, Bottino MN, Vaillancourt K, Sant'Anna GM. Implementation of a respiratory therapist-driven protocol for neonatal ventilation: impact on the premature population. Pediatrics. 2009;123:e907-16. doi:10.1542/peds.2008-1647.

24. Hummler $\mathrm{H}$, et al. Influence of different methods of synchronized mechanical ventilation on ventilation, gas exchange, patient effort, and blood pressure fluctuations in premature neonates. Pediatr Pulmonol. 1996;22:305-13. doi:10.1002/(sici)10990496(199611)22:5<305:aid-ppul3>3.0.co;2-j.

25. Greenough A, Dimitriou G, Prendergast M, Milner AD. Synchronized mechanical ventilation for respiratory support in newborn infants. Cochrane Database Syst Rev. 2008;4:CD000456. doi:10.1002/14651858.CD000456.pub3.

26. Reyes ZC, et al. Randomized, controlled trial comparing synchronized intermittent mandatory ventilation and synchronized intermittent mandatory ventilation plus pressure support in preterm infants. Pediatrics. 2006;118:1409-17. doi:10.1542/peds.2005-2923.

27. Wheeler KI, Klingenberg C, Morley CJ, Davis PG. Volume-targeted versus pressure-limited ventilation for preterm infants: a systematic review and meta-analysis. Neonatology. 2011;100:219-27. doi:10. $1159 / 000326080$.

28. Ryu J, Haddad G, Carlo WA. Clinical effectiveness and safety of permissive hypercapnia. Clin Perinatol. 2012;39:603-12. doi:10. 1016/j.clp.2012.06.001.

29. Askie LM, Henderson-Smart DJ, Irwig L, Simpson JM. Oxygensaturation targets and outcomes in extremely preterm infants. N Engl J Med. 2003;349:959-67. doi:10.1056/NEJMoa023080.

30. Supplemental therapeutic oxygen for prethreshold retinopathy of prematurity (STOP-ROP): a randomized, controlled trial. I: primary outcomes. Pediatrics 2000;105:295-10.

31. Carlo WA, et al. Target ranges of oxygen saturation in extremely preterm infants. N Engl J Med. 2010;362:1959-69. doi:10.1056/ NEJMoa0911781.

32. Stenson BJ, et al. Oxygen saturation and outcomes in preterm infants. N Engl J Med. 2013;368:2094-104. doi:10.1056/ NEJMoa1302298.

33. Schmidt B, et al. Effects of targeting higher vs lower arterial oxygen saturations on death or disability in extremely preterm infants: a randomized clinical trial. J Am Med Assoc. 2013;309:2111-20. doi:10.1001/jama.2013.5555.

34. Askie LM, et al. NeOProM: neonatal oxygenation prospective meta-analysis collaboration study protocol. BMC Pediatr. 2011;11:6. doi:10.1186/1471-2431-11-6.

35. Doyle LW, Ehrenkranz RA, Halliday HL. Dexamethasone treatment in the first week of life for preventing bronchopulmonary dysplasia in preterm infants: a systematic review. Neonatology. 2010;98:217-24. doi:10.1159/000286210.

36. Doyle LW, Ehrenkranz RA, Halliday HL. Dexamethasone treatment after the first week of life for bronchopulmonary dysplasia in preterm infants: a systematic review. Neonatology. 2010;98:289-96. doi:10.1159/000286212.

37. Watterberg KL. Policy statement-postnatal corticosteroids to prevent or treat bronchopulmonary dysplasia. Pediatrics. 2010;126:800-8. doi:10.1542/peds.2010-1534.

38. Mueller $\mathrm{M}$, et al. Predicting extubation outcome in preterm newborns: a comparison of neural networks with clinical expertise and statistical modeling. Pediatr Res. 2004;56:11-8. doi:10. 1203/01.pdr.0000129658.55746.3c.

39. Mikhno A, Ennett CM. Prediction of extubation failure for neonates with respiratory distress syndrome using the MIMIC-II clinical database. In: Annual international conference of the IEEE engineering in medicine and biology society. IEEE Engineering in Medicine and Biology Society; 2012. p. 5094-97. doi:10.1109/ embc.2012.6347139.

40. Veness-Meehan KA, Richter S, Davis JM. Pulmonary function testing prior to extubation in infants with respiratory distress syndrome. Pediatr Pulmonol. 1990;9:2-6.

41. Szymankiewicz M, Vidyasagar D, Gadzinowski J. Predictors of successful extubation of preterm low-birth-weight infants with respiratory distress syndrome. Pediatr Crit Care Med. 2005; 6:44-9.

42. Balsan MJ, Jones JG, Watchko JF, Guthrie RD. Measurements of pulmonary mechanics prior to the elective extubation of neonates. Pediatr Pulmonol. 1990;9:238-43.

43. Wilson BJ Jr, Becker MA, Linton ME, Donn SM. Spontaneous minute ventilation predicts readiness for extubation in mechanically ventilated preterm infants. J Perinatol. 1998;18:436-9.

44. Gillespie LM, White SD, Sinha SK, Donn SM. Usefulness of the minute ventilation test in predicting successful extubation in newborn infants: a randomized controlled trial. J Perinatol. 2003;23:205-7.

45. Vento G, et al. Spontaneous minute ventilation is a predictor of extubation failure in extremely-low-birth-weight infants. J Matern Fetal Neonatal Med. 2004;15:147-54.

46. Dimitriou G, Fouzas S, Vervenioti A, Tzifas S, Mantagos S. Prediction of extubation outcome in preterm infants by composite extubation indices. Pediatr Crit Care Med. 2011;12:e242-9. doi:10.1097/PCC.0b013e3181fe3431.

47. Esteban A, et al. Extubation outcome after spontaneous breathing trials with T-tube or pressure support ventilation. The Spanish Lung Failure Collaborative Group. Am J Respir Crit Care Med. 1997;156:459-65.

48. Esteban A, et al. A comparison of four methods of weaning patients from mechanical ventilation. Spanish Lung Failure Collaborative Group. N Engl J Med. 1995;332:345-50. doi:10. 1056/NEJM199502093320601.

49. Chavez A, dela Cruz R, Zaritsky A. Spontaneous breathing trial predicts successful extubation in infants and children. Pediatr Crit Care Med. 2006;7:324-8. doi:10.1097/01.pcc.0000225001. 92994.29.

50. Davis PG, Henderson-Smart DJ. Extubation from low-rate intermittent positive airways pressure versus extubation after a trial of endotracheal continuous positive airways pressure in intubated preterm infants. Cochrane Database Syst Rev. 2001;4: CD001078. doi:10.1002/14651858.CD001078. 
51. Kim EH. Successful extubation of newborn infants without preextubation trial of continuous positive airway pressure. J Perinatol. 1989;9:72-6.

52. Kim EH, Boutwell WC. Successful direct extubation of very low birth weight infants from low intermittent mandatory ventilation rate. Pediatrics. 1987;80:409-14.

53. Tapia JL, Bancalari A, Gonzalez A, Mercado ME. Does continuous positive airway pressure (CPAP) during weaning from intermittent mandatory ventilation in very low birth weight infants have risks or benefits? A controlled trial. Pediatr Pulmonol. 1995; 19:269-74.

54. Kamlin CO, Davis PG, Argus B, Mills B, Morley CJ. A trial of spontaneous breathing to determine the readiness for extubation in very low birth weight infants: a prospective evaluation. Arch Dis Child. 2008;93:F305-6. doi:10.1136/adc.2007.129890.

55. Mokhlesi B, et al. Predicting extubation failure after successful completion of a spontaneous breathing trial. Respir Care. 2007;52:1710-7.

56. Lauer M. Autonomic function and prognosis. Clevel Clin J Med. 2009;76:S18-22.

57. Lahiri MK, Kannankeril PJ, Goldberger JJ. Assessment of autonomic function in cardiovascular disease: physiological basis and prognostic implications. J Am Coll Cardiol. 2008;51:1725-33. doi:10.1016/j.jacc.2008.01.038.

58. Jubran A, Tobin MJ. Effect of isocapnic hypoxia on variational activity of breathing. Am J Respir Crit Care Med. 2000;162: $1202-9$.

59. Jubran A, Grant BJ, Tobin MJ. Effect of hyperoxic hypercapnia on variational activity of breathing. Am J Respir Crit Care Med. 1997;156:1129-39.

60. Brack T, Jubran A, Tobin MJ. Effect of resistive loading on variational activity of breathing. Am J Respir Crit Care Med. 1998; 157:1756-63.

61. Brack T, Jubran A, Tobin MJ. Effect of elastic loading on variational activity of breathing. Am J Respir Crit Care Med. 1997; 155:1341-8.

62. Bien MY, et al. Comparisons of predictive performance of breathing pattern variability measured during T-piece, automatic tube compensation, and pressure support ventilation for weaning intensive care unit patients from mechanical ventilation. Crit Care Med. 2011;39:2253-62. doi:10.1097/CCM.0b013e31822279ed.
63. Shen HN, et al. Changes of heart rate variability during ventilator weaning. Chest. 2003;123:1222-8.

64. Fairchild KD, O'Shea TM. Heart rate characteristics: physiomarkers for detection of late-onset neonatal sepsis. Clin Perinatol. 2010;37:581-98. doi:10.1016/j.clp.2010.06.002.

65. •• Kaczmarek J, et al. Heart rate variability and extubation readiness in extremely preterm infants. Neonatology. 2013;104:42-8. doi:10.1159/000347101.

66. •• Kaczmarek J, Kamlin COF, Morley CJ, Davis PG, Sant'Anna GM. Variability of respiratory parameters and extubation readiness in ventilated neonates. Arch Dis Child. 2012;98(1):F70-3. doi:10.1136/fetalneonatal-2011-301340.

67. Schmidt B, et al. Caffeine therapy for apnea of prematurity. N Engl J Med. 2006;354:2112-21. doi:10.1056/NEJMoa054065.

68. - Davis PG, et al. Caffeine for apnea of prematurity trial: benefits may vary in subgroups. J Pediatr. 2010;156:382-7. doi:10.1016/j. jpeds.2009.09.069

69. - Kirpalani $\mathrm{H}$, et al. A trial comparing noninvasive ventilation strategies in preterm infants. N Engl J Med. 2013;369:611-20. doi:10.1056/NEJMoa1214533.

70. - Yoder BA, et al. Heated, humidified high-flow nasal cannula versus nasal CPAP for respiratory support in neonates. Pediatrics. 2013;131:e1482-90. doi:10.1542/peds.2012-2742.

71. • Collins CL, Holberton JR, Barfield C, Davis PG. A randomized controlled trial to compare heated humidified high-flow nasal cannulae with nasal continuous positive airway pressure postextubation in premature infants. J Pediatr. 2013;162(949-954):e941. doi:10.1016/j.jpeds.2012.11.016.

72. Khemani RG, Randolph A, Markovitz B. Corticosteroids for the prevention and treatment of post-extubation stridor in neonates, children and adults. Cochrane Database Syst Rev. 2009;3:001000. doi:10.1002/14651858.CD001000.pub3.

73. Couser RJ, et al. Effectiveness of dexamethasone in preventing extubation failure in preterm infants at increased risk for airway edema. J Pediatr. 1992;121:591-6.

74. Overman AE, et al. Tracheostomy for infants requiring prolonged mechanical ventilation: 10 years' experience. Pediatrics. 2013;131:e1491-6. doi:10.1542/peds.2012-1943. 